\title{
Erratum to: The peritoneal dialysis catheter
}

\author{
Stefano Santarelli - Gianpaolo Amici - Tonino Bernacconi - Giuseppe Bonforte • \\ Emilio Ceraudo - Roberto Dell'Aquila • Emilio Galli • Gian Maria Iadarola • \\ Stefano Maffei - Cecilia Sardellini - Matthias Zeiler • Vincenzo La Milia • \\ Giovambattista Virga · Giuliano Brunori · Guido Garosi - Giovanni Cancarini
}

Published online: 4 September 2014

(C) Italian Society of Nephrology 2014

The correct list of authors is as shown here.

The original publication (J Nephrol. 2013 Nov-Dec;26

Suppl 21:4-75) can be found at:

http://www.sin-italy.org/web/eventi/SIN/index_rivista.cfm?

List=WsTitoloEvento,WsIdEvento\&c1 $=10 \& c 2=00187$

S. Santarelli $(\bowtie) \cdot M$. Zeiler

Nephrology and Dialysis Unit, Carlo Urbani Hospital, Jesi, Italy

e-mail: nefrologia@sin-italy.org

\author{
S. Maffei \\ Nephrology and Dialysis Unit, Maria Adelaide Hospital, Turin, \\ Italy \\ V. La Milia \\ Nephrology and Dialysis Department, A. Manzoni Hospital, \\ Lecco, Italy \\ G. Virga \\ Nephrology and Dialysis Unit, Camposampiero Hospital, \\ Camposampiero, Italy \\ G. Brunori \\ Nephrology and Dialysis Unit, Santa Chiara Hospital, \\ Trento, Italy \\ G. Garosi \\ Nephrology, Dialysis and Transplantation Unit, \\ University Hospital of Siena, Siena, Italy \\ G. Cancarini \\ Nephrology, Dialysis and Renal Transplantation Unit, \\ University Hospital Spedali Civili, Brescia, Italy
}

\section{G. Amici}

Nephrology and Dialysis Unit, S. Maria di Cà Foncello Hospital, Treviso, Italy

T. Bernacconi - C. Sardellini

Anesthesiology, Intensive Care and Analgesic Unit,

Hospital of Jesi, Jesi, Italy

G. Bonforte

Nephrology and Dialysis Unit, S. Anna Hospital, Como, Italy

E. Ceraudo

General Surgery Unit, Carlo Urbani Hospital, Jesi, Italy

R. Dell'Aquila

Nephrology and Dialysis Unit, San Bassiano Hospital,

Bassano del Grappa, Italy

E. Galli

Nephrology and Dialysis Unit, Treviglio-Caravaggio Hospital, Treviglio, Italy

G. M. Iadarola

Nephrology and Dialysis Unit, San Giovanni Bosco Hospital, Turin, Italy 